\title{
Comparison of clinical outcomes between robotic and thoracoscopic mitral valve repair
}

\author{
Shixiong Wei", Xin Zhang", Huimin Cui, Lin Zhang, Zhiyun Gong, Lianggang Li, Tong Ren, \\ Changqing Gao, Shengli Jiang \\ Department of Cardiovascular Surgery, Chinese PLA General Hospital, Beijing, China \\ Contributions: (I) Conception and design: C Gao, S Jiang; (II) Administrative support: X Zhang, L Zhang; (III) Provision of study materials or \\ patients: S Wei, L Li, T Ren; (IV) Collection and assembly of data: S Wei, H Cui; (V) Data analysis and interpretation: S Wei, Z Gong; (VI) \\ Manuscript writing: All authors; (VII) Final approval of manuscript: All authors. \\ \#These authors contributed equally to this work. \\ Correspondence to: Prof. Shengli Jiang, PhD; Prof. Changqing Gao, PhD. No. 28 Fuxing Road, Haidian Distract, Beijing 100853, China. \\ Email: jiang_shengli301@163.com; 773691039@qq.com.
}

Background: To compare the clinical outcomes and hospital cost of robotic versus thoracoscopic approaches to mitral valve plasty (MVP).

Methods: We retrospectively analyzed patients who received minimal invasive MVP between 2007 January and 2020 January at our department. The basic characteristics, echocardiography, surgical data, postoperative adverse events and hospital cost of the patients were collected. The primary outcomes of this study were direct hospital cost and 30-day outcomes, including the operative time, complications, and length of hospital stay.

Results: A total of 234 patients received minimally invasive MVP by using robotic $(\mathrm{n}=121)$ and thoracoscopic $(n=113)$ technique respectively. The overall 30 -day mortality rate was $0.9 \%(n=2)$, with no significant difference between two groups. The cardiopulmonary bypass time and aorta clamping time in thoracoscopic group were longer than that in robotic group $(153.2 \pm 25.6$ vs. $123.8 \pm 34.9$ min and $111.8 \pm 23.0$ vs. $84.9 \pm 24.3 \mathrm{~min}, \mathrm{P}<0.001)$. The intraoperative blood transfusion rate $(52.2 \%$ vs. $64.5 \%)$ and ICU time $(2.8 \pm 2.3$ vs. $3.6 \pm 2.7$ days, all $\mathrm{P}<0.05)$ of the thoracoscopic group were lower than those in the robotic group. The adjusted hospital and operating room cost of the thoracoscopic group were significant lower $(\$ 18,208.4 \pm \$ 4,429.1$ vs. $\$ 35,674.3 \pm \$ 4,936.1$ and $\$ 9,038.3 \pm \$ 2,171.7$ vs. $\$ 18,655.1 \pm \$ 2,558.3$, all $\mathrm{P}<0.001)$.

Conclusions: Both robotic and thoracoscopic approach for MVP are safe and reliable. Robotic technique has shorter operation time, while thoracoscopic technique has more advantages in blood transfusion rate, postoperative ventilation time, ICU duration and hospitalization expenses.

Keywords: Minimally invasive surgery; mitral valve repair; robotic techniques; thoracoscopic

Submitted Feb 11, 2020. Accepted for publication Aug 30, 2020.

doi: $10.21037 / \mathrm{cdt}-20-197$

View this article at: http://dx.doi.org/10.21037/cdt-20-197

\section{Introduction}

Due to excellent clinical outcomes, minimally invasive mitral valve (MV) procedure has become the preferred approach of mitral valve plasty (MVP) at certain specialized centers worldwide. This technique refers to a collection of new techniques and operation-specific technologies that directly towards less pain, shorter hospital stay, and potential cost saving (1). Adoption of minimally invasive procedure by video-assisted thoracoscopic surgery (VATS) has been gradually increasing and more recently propelled after the introduction of robotic MVP (2). Both techniques have shown excellent repair rate and durability comparable with the standard median sternotomy approach (3). 
With multiple established techniques, and few existing randomized data, controversy remains about the best approach. It has been widely accepted that a minimally invasive procedure is associated with improved perioperative outcomes, but the utility of the robotic technology remains controversial (4). Although robotic technology can provide a three-dimensional surgical field and has more flexible mechanical arms, its expensive equipment cost and complicated operation process are often considered unnecessary by some surgeons who have mastered the thoracoscopic surgery technology.

In the current era of bundled payments and value-based care, surgeons have to take both clinical outcomes and costeffectiveness of surgical procedures into account when making medical decisions. At the same time, the hospital management also has to carefully consider investments in expensive technology (4). Our institution is one of the few cardiovascular centers that gained substantial experience simultaneously in carrying out robotic and thoracoscopic cardiac surgery. In this study, we sought to evaluate the clinical outcomes and hospital cost of MVP by robotic versus thoracoscopic approach performed. Here we retrospectively analyzed our early experience with thoracoscopic approach to evaluate clinical outcomes and hospital cost and to compare these with our standard robotic approach. We present the following article in accordance with the STROBE reporting checklist (available at http://dx.doi.org/10.21037/cdt-20-197).

\section{Methods}

\section{Study population}

This is a retrospective study of patients who received minimally invasive video-assisted thoracoscopic MVP at our department between 2007 January and 2020 January. Patients were divided into two surgical groups, namely thoracoscopic MVP group $(n=113)$ and robotic MVP group ( $\mathrm{n}=121)$ according to different surgical technique, and each procedure were performed by the same surgeon (thoracoscopic MVP by Dr. S Jiang and robotic MVP by Dr. C Gao). Patients admitted to our department from January 2007 to June 2017 were treated with robotic approach, while patients admitted from September 2017 to January 2020 were treated with thoracoscopic approach. All patients were diagnosed as MV disease. Patients with concurrent procedure (coronary artery bypass grafting, congenital heart disease, aortic disease, etc.) were excluded.
The perioperative data of all patients were collected and the early outcomes ( $<30$ days) were followed up. This study was performed in accordance with the Declaration of Helsinki for human research (as revised in 2013) and was approved by our hospital's ethics committee (No. ChiCTR1900021926). The informed consent form has been signed by the patients themselves or their immediate family members before the operation.

\section{Measures of clinical outcomes and hospital cost}

Clinical effectiveness was measured by early outcomes, taking into account length of ICU stay, complications, and 30-day mortality. The primary financial outcome was operating room and total direct hospital cost, defined as the total dollar amount billed to the payor for the hospitalization. As the price level and the purchasing power of the population were different at different times, the inflation rate and the income growth of the population need to be taken into account when comparing expenses. We use the consumer price index to calculate the inflation rate, that is, inflation rate is equal to the value of a group of fixed commodities at current prices divided by the value of a group of fixed commodities at base prices and multiplied by 100 . The calculation of the increase in the income of the population takes the annual per capita wage income released by the National Bureau of Statistics as a coefficient. The adjusted medical expenses are the actual medical expenses multiplied by the inflation rate and then divided by the income growth coefficient (5).

\section{Statistical analysis}

The data were analyzed by SPSS 25.0 statistical software (SPSS, Inc., Chicago, IL, USA). Descriptive statistics were reported using means \pm standard deviations and proportions. Comparisons of data between groups were carried out by using Student's $t$-test. Counting variables are presented as frequency distribution and simple percentages. Statistical significance was set at $\mathrm{P}$ value $<0.05$.

\section{Data collection}

All blood samples were collected on an empty stomach the morning after admission. All patients underwent echocardiography examination by the same experienced ultrasound doctor within 48 hours of admission. Severe valvular regurgitation or stenosis was identified according 
to guideline recommendations (6). The surgical procedures (such as application of prosthesis, extracorporeal circulation time, aortic occlusion time, intraoperative blood loss, application of blood products, etc.) and perioperative data were recorded. The operating room cost and hospital cost were collected through the finance department of our hospital. All patients were followed up by calling or writing to themselves or their immediate family members.

\section{Results}

\section{Patient characteristics}

A total of 234 patients who were scheduled for minimally invasive MVP in our department were enrolled to this study. The patients' age was $46.5 \pm 14.0$ years, with $69.2 \%$ male (162/234). Compared with robotic MVP group, patients of thoracoscopic MVP group were older $(47.7 \pm 14.7$ $v s .45 .6 \pm 13.6)$, and had a higher ratio of cerebral infarction history (4.4\% vs. $2.5 \%$ ). While patients of robotic MVP group had a higher ratio of male $(73.6 \%$ vs. $64.6 \%)$. There was no significant difference in other risk factors and admission echocardiography between the two groups (Table 1).

\section{Perioperative outcomes}

All patients received anti-heart failure medication before, and all received selective procedure without emergency case in either subgroup. All operations were successfully performed without failure of attempted repair intraoperatively. One case of perforation of the aortic leaflet was complicated in the robotic MVP group and conversion to upper hemisternotomy was performed to repair the aortic valve. No operative death or difficulty in weaning of cardiopulmonary bypass (CPB) was noted. The CPB time and aorta clamping time of thoracoscopic MVP group were longer than that of robotic MVP group (153.2 \pm 25.6 vs. $123.8 \pm 34.9 \mathrm{~min}$ and $111.8 \pm 23.0$ vs. $84.9 \pm 24.3 \mathrm{~min}$, $\mathrm{P}<0.001)$. Difference was noted in the incidence of intraoperative transfusion between the two groups $(52.2 \%$ of thoracoscopic MVP group vs. $64.5 \%$ of robotic MVP group), and thoracoscopic MVP group had a significantly reduced postoperative ICU time than that of robotic MVP group (2.8 \pm 2.3 vs. $3.6 \pm 2.7$ days, all $\mathrm{P}<0.05$ ) (Table 2).

The overall mortality rate following operations was $0.9 \%(\mathrm{n}=2)$, with no significant difference between the two groups $[0.9 \%$ of thoracoscopic MVP group $(\mathrm{n}=1)$ vs. $0.8 \%$ of robotic MVP group (n=1) respectively]. One patient of the robotic MVP group died of severe bilateral pneumonia and respiratory distress syndrome on postoperative day 22 . And one patient of the thoracoscopic MVP group died of septic shock and multiple organ failure on postoperative day 3. Two patients in the robotic MVP group experienced transient neurological dysfunction after surgery, but all recovered smoothly before discharged. Echocardiography was performed 1 week postoperatively in all cases. In this retrospective study, all patients had none-trace mitral regurgitation immediately following operation and only three patients in robotic MVP group and one patient in thoracoscopic MVP group required mitral replacement within 30 days for early postoperative repair failure, and all achieved success.

\section{Cost comparison}

The overall initial hospital and operating room cost were $\$ 30,813.3 \pm \$ 4,178.1$ (robotic MVP group) vs. $\$ 17,155.7 \pm \$ 4,713.7$ (thoracoscopic MVP group) and $\$ 16,514.9 \pm \$ 2,278.1$ vs. $\$ 7,856.3 \pm \$ 2,500.4$ respectively. After taking into account the economic growth rate in different years, such as inflation rate, this figure has become $\$ 35,674.3 \pm \$ 4,936.1$ vs. $\$ 18,208.4 \pm \$ 4,429.1$ and $\$ 18,655.1 \pm \$ 2,558.3$ vs. $\$ 9,038.3 \pm \$ 2,171.7$ respectively. The initial and adjusted hospital and operating room cost of robotic MVP group was significantly greater than thoracoscopic MVP group (all $\mathrm{P}<0.001$ ).

\section{Discussion}

Compared with mitral valve replacement (MVR), patients who received MVP do not need long-term anticoagulation treatment after surgery, thus reducing anticoagulationrelated complications. Besides, MVP has less influence on left ventricular function due to the preservation of the native valve. Therefore, we recommend that MVP be preferred when conditions permit. MVP has conventionally been performed through median sternotomy with standard CPB. Due to resulting in an extended recovery period with substantial activity restrictions, this approach provides excellent access to the heart but challenged by the growing utility and enthusiasm for less invasive techniques first by VATS and, more recently, robotically assisted approaches in our center. Recognized as one of the most minimally invasive approach, robotic techniques have many advantages such as three-dimensional field of view, enhanced dexterity, 
Table 1 Baseline characteristics of two groups

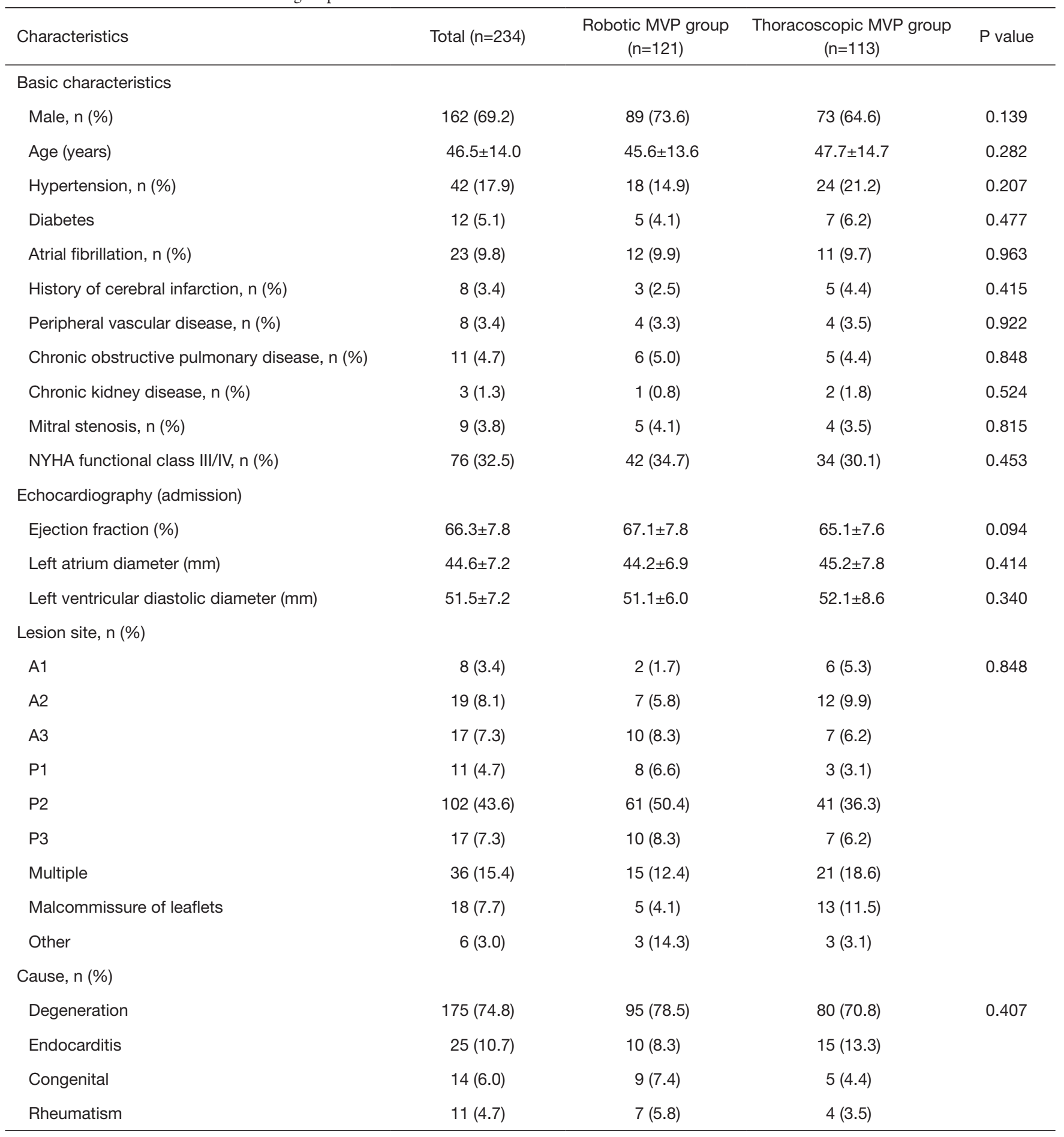

MVP, mitral valve plasty. 
Table 2 Clinical outcomes of the two groups

\begin{tabular}{|c|c|c|c|c|}
\hline Outcomes & Total $(n=234)$ & $\begin{array}{l}\text { Robotic MVP group } \\
\qquad(\mathrm{n}=121)\end{array}$ & $\begin{array}{l}\text { Thoracoscopic MVP group } \\
\qquad(n=113)\end{array}$ & $P$ value \\
\hline \multicolumn{5}{|l|}{ Operative data } \\
\hline Aorta clamping time (min) & $91.7 \pm 26.7$ & $84.9 \pm 24.3$ & $111.8 \pm 23.0$ & $<0.001^{\mathrm{a}}$ \\
\hline Intraoperative blood loss (mL) & $207.2 \pm 114.7$ & $200.8 \pm 120.2$ & $212.0 \pm 110.7$ & 0.503 \\
\hline Transfusion rate (all blood product), $\mathrm{n}(\%)$ & $137(58.5)$ & $78(64.5)$ & $59(52.2)$ & $<0.001^{\mathrm{a}}$ \\
\hline Prosthesis size (mm) & $31.0 \pm 1.8$ & $30.6 \pm 1.7$ & $31.5 \pm 1.7$ & $<0.001^{\mathrm{a}}$ \\
\hline ICU time (days) & $3.3 \pm 2.6$ & $3.6 \pm 2.7$ & $2.8 \pm 2.3$ & $0.031^{a}$ \\
\hline Atrial septal defect repair & $5(2.2)$ & $3(2.5)$ & $2(1.8)$ & $<0.001^{\mathrm{a}}$ \\
\hline Patent foramen ovale ligation & $2(0.9)$ & $2(1.7)$ & 0 & \\
\hline Atrial myxoma resection & $3(1.3)$ & $1(0.8)$ & $2(1.8)$ & \\
\hline Left atrial appendage ligation & $3(1.3)$ & 0 & $3(2.7)$ & \\
\hline Tricuspid valve procedure & $32(13.7)$ & 0 & $32(33.3)$ & \\
\hline \multicolumn{5}{|l|}{ Complications, n (\%) } \\
\hline Conversion to sternotomy & $1(0.4)$ & $1(0.8)$ & 0 & 0.335 \\
\hline Pleural effusion with pleurocentesis & $3(1.3)$ & $2(1.7)$ & $1(0.9)$ & 0.604 \\
\hline \multicolumn{5}{|l|}{ Postoperative echocardiography (1 week) } \\
\hline Ejection fraction (\%) & $60.6 \pm 6.9$ & $61.9 \pm 6.1$ & $58.8 \pm 7.7$ & 0.004 \\
\hline Left atrium diameter (mm) & $34.2 \pm 6.2$ & $34.0 \pm 6.3$ & $34.3 \pm 6.1$ & 0.747 \\
\hline Left ventricular diastolic diameter (mm) & $43.6 \pm 5.9$ & $43.7 \pm 6.3$ & $43.5 \pm 5.3$ & 0.788 \\
\hline \multicolumn{5}{|l|}{ Cost (\$) } \\
\hline Operating room cost & $12,810.2 \pm 2,506.3$ & $16,514.9 \pm 2,278.1$ & $7,856.3 \pm 2,500.4$ & $<0.001^{\mathrm{a}}$ \\
\hline Hospital cost & $26,258.8 \pm 4,758.6$ & $30,813.3 \pm 4,178.1$ & $17,155.7 \pm 4,713.7$ & $<0.001^{\mathrm{a}}$ \\
\hline Adjusted operating room cost & $14,974.3 \pm 2,711.9$ & $18,655.1 \pm 2,558.3$ & $9,038.3 \pm 2,171.7$ & $<0.001^{\mathrm{a}}$ \\
\hline Adjusted hospital cost & $29,731.9 \pm 5,811.7$ & $35,674.3 \pm 4,936.1$ & $18,208.4 \pm 4,429.1$ & $<0.001^{\mathrm{a}}$ \\
\hline
\end{tabular}

\footnotetext{
${ }^{\mathrm{a}}, \mathrm{P}<0.05 . \mathrm{MVP}$, mitral valve plasty.
} 
Table 3 Surgical technique of the two groups

\begin{tabular}{lccc}
\hline Techniques & Robotic MVP group $(\mathrm{n}=121)$, & Thoracoscopic MVP group $(\mathrm{n}=113)$, & $\mathrm{T}(\%)$ \\
\hline Annuloplasty ring & $\mathrm{n}(\%)$ & $111(98.2)$ & $4(1.7)$ \\
Artificial chordae tendineae implantation & $102(84.3)$ & $67(59.3)$ & $87(37.2)$ \\
Leaflet resection & $20(16.5)$ & $6(5.3)$ & $18(15.9)$ \\
Commissural magic stitch & $79(65.3)$ & $5(4.4)$ & $23(9.8)$ \\
Congenital crack suture & $5(4.1)$ & $5(4.4)$ & $14(6.0)$ \\
Commissurotomy & $9(7.4)$ & $2(1.8)$ & $7(5.1)$ \\
Papillary muscle mass resection & $7(5.8)$ & $7(6.2)$ & $7(3.0)$ \\
Other & 0 & 0 & $0.8)$ \\
\hline
\end{tabular}

"Other" represents surgical techniques that do not belong to the above classification, including Barlow's disease correction, etc. MVP, mitral valve plasty.

tremor filtration, and telesurgical capabilities (7). But at the same time, we must realize the reality that in the current climate of health economics, the sustainability of new technology is often predicated on the demonstration of financial viability and responsible resource utilization (8).

The results of this study showed excellent outcomes of both robotic and thoracoscopic techniques with low incidence of morbidity and mortality. With no significant difference between two groups on preoperative characteristics, the perioperative results revealed that patients in thoracoscopic MVP group has more advantages in intraoperative blood transfusion rate, postoperative ventilation time, ICU stay and hospitalization expenses. While patients in robotic MVP group had shorter CPB time and aorta clamping time. Besides, although there were no statistically significant differences, a trend toward less intraoperative blood loss and 24-hour drainage volume were observed with the robotic MVP group and the thoracoscopic MVP group respectively. Patients in the thoracoscopic MVP group received more annuloplasty band implantation $(98.2 \%$ vs. $84.3 \%)$, artificial chordae tendineae implantation $(59.3 \%$ vs. $16.5 \%)$ and tricuspid valve procedure $(33.3 \%$ vs. $0 \%$, all $\mathrm{P}<0.001)$ than robotic MVP group. We believed that the difference in operation duration between the two groups was mainly caused by the different surgical techniques applied and the early extubation strategy advocated in recent years (Table 3). We also noticed that U-Clip sutures were applied more in the robotic MVP group.

The greater precision afforded by the robotic approach compared with thoracoscopic approach has fueled our interest in comparative studies to formally evaluate the two techniques particularly in light of the increased resource allocation necessary in the establishment of a robotic program $(9,10)$. Results have unsurprisingly confirmed increased costs involved in the establishment of the latter. Whether inflation rate and per capita income increase were taken into account or not, the hospitalization expenses of patients in the robotic MVP group were significantly higher than those in the thoracoscopic MVP group.

Although MV repair procedure may be a likely potential avenue in which robotic techniques will maintain an edge over VATS, according to our experience, robotic techniques are more difficult than thoracoscopic procedures and a learning curve is necessary to be faced with telemanipulation technology. Surgeons experienced in minimally invasive MVP procedure may find this learning curve easier than others. This study showed that the operation time of robotic MVP was shorter than that of thoracoscopic approach, but it was worth noting that our department had carried out robot surgery in 2007 , but it would only carry out thoracoscopic surgery in 2016. Besides, concerns of quality must be respected, especially when dealing with a largely elective, low risk population. Thus, surgeons unable to perform minimally invasive non-robotic mitral repair with similar outcomes to sternotomy are advised to consider additional training prior to embarking on a full switch to a robotic platform (11).

\section{Limitation}

This study has several limitations. As a retrospective cohort 
study, an inherent selection bias may exist that cannot be fully eliminated. Power to detect significant differences was limited by the small sample size in each group. The specific costs cited in our study may not be applicable to other institutions. Furthermore, as a single center experience, these results may not be generalizable to other settings. This subject will need further analysis, because more data are accrued from well-designed prospective studies in the future. Meanwhile, the mid-term and long-term outcomes of the two groups also need to be confirmed by follow-up.

\section{Conclusions}

Reasonable evidence exists to support the fact that, as minimally invasive procedures, both robotic and thoracoscopic MVP techniques result in safe and reliable early outcomes. Robotic procedure has shorter operation time, while thoracoscopic procedure has more advantages in postoperative ventilation time, ICU duration, intraoperative blood transfusion rate and hospitalization expenses.

\section{Acknowledgments}

Funding: None.

\section{Footnote}

Reporting Checklist: The authors present the study in accordance with the STROBE reporting checklist. Available at http://dx.doi.org/10.21037/cdt-20-197

Data Sharing Statement: Available at http://dx.doi. org/10.21037/cdt-20-197

Peer Review File: Available at http://dx.doi.org/10.21037/ cdt-20-197

Conflicts of Interest: All authors have completed the ICMJE uniform disclosure form (available at http://dx.doi. org/10.21037/cdt-20-197). The authors have no conflicts of interest to declare.

Ethical Statement: The authors are accountable for all aspects of the work in ensuring that questions related to the accuracy or integrity of any part of the work are appropriately investigated and resolved. This study was performed in accordance with the Declaration of Helsinki for human research (as revised in 2013) and was approved by our hos- pital's ethics committee (No. ChiCTR1900021926). The informed consent form has been signed by the patients themselves or their immediate family members before the operation.

Open Access Statement: This is an Open Access article distributed in accordance with the Creative Commons Attribution-NonCommercial-NoDerivs 4.0 International License (CC BY-NC-ND 4.0), which permits the noncommercial replication and distribution of the article with the strict proviso that no changes or edits are made and the original work is properly cited (including links to both the formal publication through the relevant DOI and the license). See: https://creativecommons.org/licenses/by-nc-nd/4.0/.

\section{References}

1. Modi P, Rodriguez E, Hargrove WC 3rd, et al. Minimally invasive video-assisted mitral valve surgery: a 12 -year, 2-center experience in 1178 patients. J Thorac Cardiovasc Surg 2009;137:1481-7.

2. Kneuertz PJ, Singer E, D'Souza DM, et al. Hospital cost and clinical effectiveness of robotic-assisted versus videoassisted thoracoscopic and open lobectomy: A propensity score-weighted comparison. J Thorac Cardiovasc Surg 2019;157:2018-26.e2.

3. Kesävuori R, Raivio P, Jokinen JJ, et al. Early experience with robotic mitral valve repair with intra-aortic occlusion. J Thorac Cardiovasc Surg 2018;155:1463-71.

4. Long H, Tan Q, Luo Q, et al. Thoracoscopic Surgery Versus Thoracotomy for Lung Cancer: Short-Term Outcomes of a Randomized Trial. Ann Thorac Surg 2018;105:386-92.

5. D'Amico TA. Undoing the gaps in quality, cost, and value. J Thorac Cardiovasc Surg 2018;155:1211.

6. Hahn RT, Abraham T, Adams MS, et al. Guidelines for performing a comprehensive transesophageal echocardiographic examination: recommendations from the American Society of Echocardiography and the Society of Cardiovascular Anesthesiologists. J Am Soc Echocardiogr 2013;26:921-64.

7. Xu H, Li J, Sun Y, et al. Robotic versus laparoscopic right colectomy: a meta-analysis. World J Surg Oncol 2014;12:274.

8. Chen CW, Atluri P. Robotic mitral valve surgery: Additive benefits without additive cost. J Thorac Cardiovasc Surg 2018;156:1038-9.

9. Deen SA, Wilson JL, Wilshire CL, et al. Defining the cost 
of care for lobectomy and segmentectomy: a comparison of open, video-assisted thoracoscopic, and robotic approaches. Ann Thorac Surg 2014;97:1000-7.

10. Swanson SJ, Miller DL, McKenna RJ Jr, et al. Comparing robot-assisted thoracic surgical lobectomy with conventional video-assisted thoracic surgical lobectomy

Cite this article as: Wei S, Zhang X, Cui H, Zhang L, Gong Z, Li L, Ren T, Gao C, Jiang S. Comparison of clinical outcomes between robotic and thoracoscopic mitral valve repair. Cardiovasc Diagn Ther 2020;10(5):1167-1174. doi: 10.21037/ cdt-20-197 and wedge resection: results from a multihospital database (Premier). J Thorac Cardiovasc Surg 2014;147:929-37.

11. Valdis M, Chu MW, Schlachta C, et al. Evaluation of robotic cardiac surgery simulation training: A randomized controlled trial. J Thorac Cardiovasc Surg 2016;151:1498505.e2. 